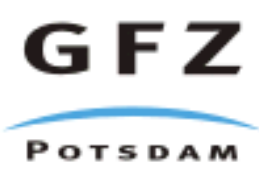

Originally published as:

Jahn, S., Ollivier, J., Demmel, F. (2008): Fast ionic mobility in cryolite studied by quasielastic neutron scattering. - Solid State Ionics, 179, 35-36, 1957-1961

DOI: $10.1016 /$ j.ssi.2008.06.006 


\title{
Fast ionic mobility in cryolite studied by quasielastic neutron scattering
}

\author{
Sandro Jahn \\ GeoForschungsZentrum Potsdam, Section 4.1, Telegrafenberg, 14473 Potsdam, \\ Germany \\ Jacques Ollivier \\ Institut Laue-Langevin, BP 156, 38042 Grenoble Cedex 9, France \\ Franz Demmel \\ ISIS Facility, Chilton, OX11 0QX, United Kingdom
}

\begin{abstract}
Quasielastic neutron scattering (QENS) has been used to study the relation between ionic mobility and conductivity at high temperature of the perovskite fluoride cryolite, $\mathrm{Na}_{3} \mathrm{AlF}_{6}$. Up to $T=880{ }^{\circ} \mathrm{C}$ the conductivity is dominated by jump diffusion of Na ions. At higher temperatures, a considerable broadening of the QENS spectra and the development of a liquid-like diffraction peak is observed. The $Q-$ dependence of the line width suggests a set-in of translational diffusion of fluorines at this temperature. This additional degree of translational movements could reason the jump-like increase in the ionic conductivity observed macroscopically.
\end{abstract}

Key words: Cryolite; $\mathrm{Na}_{3} \mathrm{AlF}_{6}$; Quasielastic neutron scattering; Ionic conduction; Self-diffusion

\section{Introduction}

Materials with a perovskite-type structure are of great interest in many fields of science and technology. Their applications range from the use as catalysts or sensors to superconductors or ferroelectric materials. In Earth sciences, the

* Corresponding author.

Email address: jahn@gfz-potsdam.de (Sandro Jahn). 
most important perovskite structure is of $(\mathrm{Mg}, \mathrm{Fe}) \mathrm{SiO}_{3}$ composition, which is thought to be the major component of the Earth's lower mantle. Despite the structural similarities, the specific behavior of different perovskites varies substantially. A number of perovskites are characterized by a high ionic mobility, especially at high temperatures, $T$. The understanding of ionic conduction mechanisms in perovskites has been subject to a number of experimental and simulation studies (see [1] for a recent review).

For fluoride perovskites, vacancy mechanisms for ionic diffusion have been proposed using results from conductivity and nuclear magnetic resonance (NMR) measurements [2], neutron diffraction experiments [3,4] or molecular dynamics computer simulations [5]. Diffusion rates may be obtained from NMR data $[2,6,7]$, but the sensitivity of NMR is usually limited to jump frequencies up to the $\mathrm{kHz}$ range. For faster ionic mobility, experimental data are rare due to experimental difficulties of performing in situ experiments at high $T$ where fluoride samples often become very reactive. In this study, we investigate the microscopic mechanisms and rates of fast ionic diffusion in a model perovskite (cryolite) using an alternative experimental technique, quasielastic neutron scattering (QENS).

Cryolite, $\mathrm{Na}_{3} \mathrm{AlF}_{6}$, is a rare mineral mainly identified with the formerly large deposit of Ivigtut, Greenland. It is used in industry as a flux to extract aluminum metal from bauxite. In modern technological processes the mineral is replaced by synthetic sodium aluminum fluoride. Besides these industrial applications cryolite is a model compound of a mixed-cation perovskite $\left(\mathrm{Na}_{2}(\mathrm{NaAl}) \mathrm{F}_{6}\right)$. In the crystal structure, all of the $\mathrm{Al}^{3+}$ and one third of the $\mathrm{Na}^{+}$cations are six-fold coordinated $\left(\mathrm{Na}^{V I}\right)$ in the center of alternating $\left[\mathrm{AlF}_{6}\right]$ and $\left[\mathrm{NaF}_{6}\right]$ octahedra, whereas the remaining $\mathrm{Na}^{+}$ions are eight-fold coordinated $\left(\mathrm{Na}^{V I I I}\right)$. At low temperatures, cryolite has a monoclinic crystal structure $\left(\mathrm{P} 2_{1} / \mathrm{n}\right)$. Several high temperature $\mathrm{x}$-ray diffraction experiments showed a transition from the monoclinic to a cubic $(F m \overline{3} m)[8,9]$ or orthorhombic $(I m m m)$ [10] structure around $T=567{ }^{\circ} \mathrm{C}$. This phase transition is accompanied by a sudden increase in the electrical conductivity. A second jump in the conductivity is observed at $T=880{ }^{\circ} \mathrm{C}$, well below the melting point $\left(T_{m}=1013{ }^{\circ} \mathrm{C}\right)[11]$. At this temperature, an eutectic is observed on the $\mathrm{NaF}$-rich side of the $\mathrm{NaF}-\mathrm{AlF}_{3}$ phase diagram [12] and hence a partial melt is expected to develop.

Results from NMR spectroscopy indicate an increased ionic mobility already above $T=100{ }^{\circ} \mathrm{C}$, well within the stability range of the monoclinic phase $[6,7]$. The observed changes in the chemical shift and the merging of the two peaks from the differently coordinated $\mathrm{Na}$ ions are interpreted as rapid cation exchange between the $\mathrm{Na}^{V I}$ and $\mathrm{Na}^{V I I I}$ sites. At $\mathrm{T}=600{ }^{\circ} \mathrm{C}$, which is above the phase transition to the cubic phase, the two sides are indistinguishable on the time scale of NMR with a minimum exchange frequency of $13 \mathrm{kHz}$. It 
was proposed $[6,7]$ that the $\mathrm{Na}$ diffusion is governed by a vacancy mechanism and the respective diffusion rate is controlled by oscillations of the $\left[\mathrm{AlF}_{6}\right]$ octahedra. Molecular dynamics simulations suggest that the $\left[\mathrm{AlF}_{6}\right]$ octahedra rotate above $T=300{ }^{\circ} \mathrm{C}$ [13]. Whereas $\mathrm{Na}$ diffusion in stoichiometric cryolite was not observed in the simulations below $T=800{ }^{\circ} \mathrm{C}$, this temperature is considerably reduced when defects (vacancies) are present [14]. This is also consistent with thermodynamic data that suggest non-stoichiometric cryolite at elevated temperatures [12].

The present study is concerned with diffusion processes at temperatures well above the monoclinic to cubic phase transition up to the melting point. Especially the region around the second jump of the conductivity at $T=880{ }^{\circ} \mathrm{C}$ [11] has not been studied by means of microscopic methods, such as diffraction or spectroscopy. The QENS technique is a powerful tool to investigate the elementary steps of diffusion on atomic length $\left(10^{-10}\right.$ to $10^{-9}$ meters $)$ and time $\left(10^{-12}\right.$ to $10^{-9}$ seconds) scales. QENS experiments can be performed in situ at high temperatures. The measured spectral function, the so called dynamic structure factor $S(Q, \omega)$, represents the Fourier transform of the time dependent atomic density correlations in the sample. The spectral width in frequency, $\omega$, space is inversely proportional to the decay time of the correlation function. Hence, it provides access to the time scales involved in the diffusion processes. The momentum transfer, $Q$, dependence of the spectral width delivers information about the geometry of the movements.

\section{Experimental Section}

The natural sample from the Ivigtut area, Greenland, was obtained from the mineral collection of the Naturkundemuseum Berlin that is associated with the Humboldt University Berlin, Germany. In addition, a synthetic sample from Testbourne Limited (99.995 \% pure) was used. Both samples were crushed into powder. Refinement of x-ray powder diffraction data confirmed the presence of a single crystalline phase (cryolite) for the synthetic sample whereas the natural sample consists of at least $98 \%$ cryolite and a small content of quartz. Both powder samples were enclosed in molybdenum cylindrical containers with a wall thickness of $0.2 \mathrm{~mm}$ and an inner diameter of $8 \mathrm{~mm}$. After filling the containers in inert atmosphere they were sealed by electron beam welding.

QENS experiments were performed at the cold neutron time-of-flight spectrometer IN5 of the Institut Laue-Langevin (ILL) Grenoble, France. QENS spectra were taken at various temperatures between room temperature up to $T=1080{ }^{\circ} \mathrm{C}$, just above the melting point. Two wavelengths of the incident monochromatic neutron beam were chosen to compromise between resolution and the kinematically accessible range of energy transfers, $\hbar \omega(\hbar=h / 2 \pi, h$ 
is Planck's constant). With $\lambda=7.6 \AA$ and $\lambda=5.0 \AA$, energy resolutions of $29 \mu \mathrm{eV}$ and $100 \mu \mathrm{eV}$ were obtained, respectively. The corresponding ranges of momentum transfer, $Q$, were $0.3-1.5 \AA^{-1}$ and $0.4-2.2 \AA^{-1}$. For background correction and data normalization, spectra of an empty container and a vanadium spiral with dimensions and scattering power similar to the sample were taken, respectively. All spectra with $\lambda=7.6 \AA$ were obtained during heating and spectra with $\lambda=5.0 \AA$ during cooling of the samples, respectively.

Corrections to the raw data include subtraction of the background, corrections for the self-absorption of the sample and detector efficiency. Contributions from multiple scattering are expected to be much broader in energy than the QENS signal. Intensity from multiphonon scattering is weak at small momentum transfers and is supposedly unstructured flat in the considered energy transfer ranges near the elastic line. Both contributions are accounted for by a flat background in the fitting procedure. Finally, the corrected time-of-flight spectra, $S(2 \theta, \omega)$, taken at constant scattering angles, $\theta$, are transformed into the dynamic structure factor $S(Q, \omega)$ by spline fits of $S(2 \theta, \omega)$ at constant $Q$. Details of the data treatment are given elsewhere [15].

\section{Results}

The temperature dependence of the quasielastic spectra is demonstrated in cuts of $S(Q, \omega)$ at constant $Q, Q=1.0 \AA^{-1}$ (Fig. 1) and $Q=2.0 \AA^{-1}$ (Fig. 2). Below $T=600{ }^{\circ} \mathrm{C}$, the spectra are dominated by an elastic line centered at $\hbar \omega=0$ and broadened by the resolution function, and inelastic contributions from vibrational motions. Negative $\omega$ refer to neutron energy gain data, positive $\omega$ to neutron energy loss data, respectively. Vibrational modes are clearly visible in Fig. 2 between 5 and $15 \mathrm{meV}$. The spectrum at $T=600{ }^{\circ} \mathrm{C}$ in Fig. 2 still shows some flat inelastic scattering at lower energy transfers, which is probably due to incomplete structural relaxation. At higher temperatures, spectra taken during heating and cooling are essentially the same.

Substantial broadening of the quasielastic line starts around $800{ }^{\circ} \mathrm{C}$. A dramatic change in the line width occurs between $850{ }^{\circ} \mathrm{C}$ and $900{ }^{\circ} \mathrm{C}$, which corresponds to the temperature range of the second jump in the conductivity at $880{ }^{\circ} \mathrm{C}$ [11]. There is no significant difference in the spectra between 900 and $970{ }^{\circ} \mathrm{C}$. By crossing the melting point $\left(T_{m}=1013{ }^{\circ} \mathrm{C}\right)$ a surprisingly small change in the dynamics has been observed, only influencing the relative intensities of the two Lorentzians.

The change of the atomic dynamics around $880{ }^{\circ} \mathrm{C}$ is accompanied by structural changes. Due to the lack of diffraction data at these elevated temperatures, we use the dynamic data available to extract structural information. 
Integration of $S(Q, \omega)$ over the measured range of $\hbar \omega$ yields a good estimate of the total static structure factor, $S(Q)$. In the solid state $\left(T<880^{\circ} \mathrm{C}\right)$, a crystal powder diffraction line is observed around $Q=1.3 \AA^{-1}$, which disappears during the transition at $880{ }^{\circ} \mathrm{C}$. Instead, a broad structural peak emerges in the same $Q$ range (see Fig. 3), which is a clear sign of structural disorder as observed, e.g., in melts and glasses.

\section{Discussion}

The total dynamic structure factor, $S(Q, \omega)$, is composed of partial contributions from incoherent and coherent scattering $(i, j=\mathrm{Na}, \mathrm{Al}, \mathrm{F})$

$$
\begin{aligned}
\frac{\sigma_{B}}{4 \pi} S(Q, \omega) & =\sum_{i} c_{i} \frac{\sigma_{i n c}^{i}}{4 \pi} S_{s}^{i}(Q, \omega) \\
& +\sum_{i} \sum_{j} \sqrt{c_{i} c_{j}} b_{c o h}^{i} b_{c o h}^{j} S^{i j}(Q, \omega)
\end{aligned}
$$

with $\sigma_{B}$ being the total bound neutron scattering cross-section of the sample, $\sigma_{\text {inc }}$ and $b_{c o h}^{i}$ the incoherent cross-section and the coherent scattering length of species $i . c_{i}$ are the corresponding concentrations. $S^{i j}(Q, \omega)$ are the partial coherent dynamic structure factors and $S_{s}^{i}(Q, \omega)$ the respective incoherent structure factor, i.e. the double Fourier transform of the self-correlation functions. Integration over $\hbar \omega$ yields a similar equation for $S(Q)$.

Using the values given in Tab. 1, weighting factors for the different partials are readily calculated. They indicate the relative contribution of the partials to the total $S(Q, \omega)$. While sodium is the dominant incoherent scatterer, significant signals from coherent scattering should always include fluorine correlations. Integrating the total $S(Q, \omega)$ over $\hbar \omega$ at low temperatures (disregarding Bragg peaks) leads to total static structure factors that are essentially constant, i. e. that do not depend on $Q$, and that represent the incoherent structure factor $S_{s}^{N a}(Q)=$ constant of the Na atoms. Since the experimental $S(Q, \omega)$ is normalized according to Eq. 1 using the cross-sections of Tab. 1, the expected amplitude of $S_{s}^{N a}(Q)$ is $c_{N a} \sigma_{i n c}^{N a} / \sigma_{B}=0.14$.

The integrated $S(Q, \omega)$ at low temperatures is about 0.15 , which is consistent with the theoretical value during heating (see e.g. $S_{1}(Q)$ for $T=700{ }^{\circ} \mathrm{C}$ ) and confirms the performed absolute normalization. It increases to about 0.2 after melting the sample (see Fig. 3), which is readily explained by the compaction of the sample during melting and hence an increase of scatterers in the neutron beam. For the normalization we used an effective density for the powder of $67 \%$ of the bulk density of cryolite $\left(2.97 \mathrm{~g} / \mathrm{cm}^{3}\right)$, which seems a reasonable 
approximation. It is expected that the sample is more compact when it recrystallizes from the melt during cooling. We do not make an attempt to correct for the change in density since this should have no effect on the dynamics but the uncertainty in effective density should be borne in mind when discussing absolute amplitudes at different temperatures.

In addition to the plateau in $S(Q)$ at low temperatures, a $Q$-dependent structural peak of correlations that include fluorine (F-F, F-Na, F-Al) appears at temperatures above $800{ }^{\circ} \mathrm{C}$.

With a simple diffusion model, spectra can be described by a Lorentzian line with $Q$-dependent line width $\Gamma(Q)$ :

$$
S(Q, \omega)=\frac{S(Q)}{\pi} \frac{\Gamma(Q)}{(\hbar \omega)^{2}+\Gamma(Q)^{2}}
$$

At small $Q$ vectors, $\Gamma$ is expected to be proportional to $D Q^{2}$. The proportionality constant $D$ is the self-diffusion coefficient for translational diffusion on long distances. This process is catalyzed by thermal energy and the diffusion constant D should follow an Arrhenius law. The amplitude of the Lorentzian $S(Q)$ should be constant in $Q$ if only incoherent scattering occurs.

Up to $T \sim 800{ }^{\circ} \mathrm{C}$, just before the QENS lines start to broaden, a single Lorentzian is sufficient to fit our data. At higher temperatures, a second Lorentzian has to be added, which implies that additional dynamic processes come into play. In Fig. 4, fits of the measured spectra at $T=850{ }^{\circ} \mathrm{C}$ $(\lambda=7.6 \AA)$ and at $T=970{ }^{\circ} \mathrm{C}(\lambda=5.0 \AA)$ are shown for a $Q$-vector magnitude of $1.0 \AA^{-1}$. In the fitting procedure, the two Lorentzians are convoluted with the respective resolution function of the spectrometer. The spectra are composed of a sharp line on top of a broad line. In Fig. 5 the resulting fit parameters, the widths $\Gamma(Q)$ and amplitudes $S(Q)$ of the two Lorentzians, are depicted.

Below $900{ }^{\circ} \mathrm{C}, S_{1}(Q)$ is the amplitude of the narrow line and shows a weak decrease with rising $Q$-vectors, which is expected for an incoherent scatterer performing translational movements. The increase of the line width $\Gamma_{1}$ in Fig. $5 b$ indicates a translational diffusion process. This motion can be attributed to sodium ions. The second (broad) line has an amplitude $S_{2}(Q)$ which exhibits a completely different $Q$ behavior, increasing towards the $Q$-vector of the DebyeScherrer line at $Q=1.3 \AA^{-1}$. This is expected from a signal based on coherent scattering. We attribute it to coherent scattering of mainly fluorines, but also partly from the sodium coherent scattering cross section. Due to the small amplitude of $S_{2}(Q)$ below $T=850{ }^{\circ} \mathrm{C}$, the $Q$-dependence of the corresponding width $\Gamma_{2}$ has a rather high level of uncertainty. A line width independent of $Q$ would indicate a fast local motion, i.e. a rotation of $\mathrm{AlF}_{6}$ octahedra could be a 
possible movement to explain the broad line. At higher temperature, however, $\Gamma_{2}$ shows a clear $Q$ dependence. Hence, the possibility of a simple rotation mechanism of F motion is unlikely, since this would require a $Q$-independent evolution of the line width [16]. Taking into account the assumption, that mainly fluorine correlations contribute to the coherent structure factor, we come to the conclusion that fluorine diffusion sets in at least at $T=880{ }^{\circ} \mathrm{C}$, whereas the fluorine dynamics at lower temperatures could still be dominated by rotations.

Crossing the eutectic temperature the fit parameters of the first Lorentzian change dramatically. The line widths of both contributions show a broadening with rising $Q$ vector, indicating translational movements. Both motions appear on a similar time scale. Furthermore the amplitudes depict a $Q$-dependence related to coherent correlations. A separate assignment of the two Lorentzians to the sodium motion and the fluorine motion is not possible anymore. The narrow contribution from $\mathrm{Na}$ incoherent scattering is replaced by a very broad, liquid-like spectral feature that probably contains components of sodium and fluorine diffusion. For both spectral components we suppose a partial contribution from the sodium and the fluorine ions.

If the microscopic interpretation of the QENS spectra given above is correct it should be possible to explain the features observed in macroscopic conductivity measurements [11]. In the following, we therefore concentrate on the low $Q$ range where a $D Q^{2}$ dependence of the line widths can be assumed. Diffusion coefficients have been extracted by fitting a $D Q^{2}$ function to the line widths at small $Q$ up to about $Q=0.6 \AA^{-1}$, as indicated in Fig. 5. The respective self-diffusion coefficients are shown in Fig. 6. The derived diffusion coefficients show almost Arrhenius-type behavior. Interestingly, no jump is observed at the melting point.

The Nernst-Einstein equation is used to obtain ionic conductivities, $\sigma$, from the self-diffusion coefficients:

$$
\sigma=\frac{e^{2} n}{k_{B} T} \sum_{i} z_{i}^{2} c_{i} D_{\sigma, i}
$$

with $e$ being the electron charge, $n$ is the particle density and $k_{B}$ Boltzmann's constant. The sum includes the charge $z_{i}(\mathrm{Na}:+1, \mathrm{Al}:+3, \mathrm{~F}:-1)$, the concentration $c_{i}$ and the conductivity diffusion coefficient $D_{\sigma, i}$ of all mobile ionic species $i$. Due to blocking and correlation effects, $D_{\sigma, i}$ is expected to be smaller than the corresponding self-diffusion coefficient. The respective scaling factor depends on the concentration of mobile species and the availability of empty sites for jump diffusion [16].

Using equation 3, an estimate of the partial contributions to the ionic con- 
ductivity is calculated. For the concentrations it is assumed that the narrow quasielastic line represents only $\mathrm{Na}$ as a diffusive species. Above $880{ }^{\circ} \mathrm{C}$, we consider both $\mathrm{F}$ and $\mathrm{Na}$ as mobile species, which equally contribute to the two broad lines. This assumption was made since $S_{1}(Q)$ and $S_{2}(Q)$ are of similar magnitude at high temperatures.

As expected, the calculated conductivity obtained from setting $D_{\sigma, i}=D_{i}$ is larger than the actual electrical conductivity measured by Landon and Ubbelohde [11] (Fig. 7). For $T<880{ }^{\circ} \mathrm{C}$ and for temperatures above the melting point, a blocking factor of 0.2 can be derived to get an agreement between $D_{i}$ and $\sigma$. Furthermore, the slopes of the conductivity curves obtained from the Nernst-Einstein model are in excellent agreement with the conductivity data from [11] below $T=880{ }^{\circ} \mathrm{C}$ and above the melting point. Hence, the model assumptions seem to be valid in these two temperature ranges and the QENS spectra capture essential contributions of the ionic conductivity. The strong increase in electrical conductivity [11] just at the eutectic temperature $\left(T=880{ }^{\circ} \mathrm{C}\right)$ might be linked to the appearance of a broad contribution in the QENS spectra and hence to the onset of translational fluorine mobility in the QENS time window.

In conclusion, we have confirmed that below $880{ }^{\circ} \mathrm{C}$ the dominant contribution to the ionic conductivity in cryolite is due to the diffusion of $\mathrm{Na}$ ions. $\mathrm{AlF}_{n}$ polyhedra with $n \leq 6$ are expected to rotate but fluorines may also have started to diffuse by jumping between different polyhedra, a supposition, already put forward by Landon and Ubbelohde [11]. The jump in the electrical conductivity at the eutectic temperature is probably caused by the strong increase in fluorine mobility. Surprisingly small changes in the atomic dynamics are observed across the melting point. Further experimental and modeling studies are needed to fully understand the diffusion mechanisms in the high temperature solid.

\section{Acknowledgment}

We would like to acknowledge the Naturkundemuseum Berlin for providing the natural sample. It is a pleasure to thank Steve Jenkins for the help during the start-up of the experiment.

\section{References}

[1] S. Hull, Rep. Prog. Phys. 67 (2004) 1233. 
[2] A. V. Chadwick, J. H. Strange, G. A. Ranieri, M. Terenzi, Solid State Ionics 9-10 (1983) 555 .

[3] C. Ridou, M. Rousseau, B. Pernot, J. Bouillot, J. Phys. C: Solid State Phys. 19 (1986) 4847.

[4] D. Z. Demetriou, C. R. A. Catlow, A. V. Chadwick, G. J. McIntyre, I. Abrahams, Solid State Ionics 176 (2005) 1571.

[5] G. W. Watson, S. C. Parker, A. Wall, J. Phys.: Condens. Matter 4 (1992) 2097.

[6] D. R. Spearing, J. F. Stebbins, I. Farnan, Phys. Chem. Minerals 21 (1994) 373.

[7] M. Kotecha, S. Chaudhuri, C. P. Grey, L. Frydman, J. Am. Chem. Soc. 127 (2005) 16701.

[8] E. G. Steward, H. P. Rooksby, Acta Cryst. 6 (1953) 49.

[9] Q. Zhou, B. J. Kennedy, J. Sol. State Chem. 177 (2004) 654.

[10] H. Yang, S. Ghose, D. M. Hatch, Phys. Chem. Minerals 19 (1993) 528.

[11] G. J. Landon, A. R. Ubbelohde, Proc. Roy. Soc. Lond. 240 (1957) 160.

[12] E. W. Dewing, Metall. Mat. Trans. B 28 (1997) 1095.

[13] M. J. Castiglione, Ph.D. thesis, University of Oxford (2000).

[14] L. Foy, P. A. Madden, J. Phys. Chem. B 110 (2006) 15302.

[15] S. Jahn, Ph.D. thesis, TU Chemnitz (2003).

[16] R. Hempelmann, Quasielastic neutron scattering and solid state diffusion, Clarendon Press, Oxford, 2000.

[17] V. F. Sears, Neutron News 3 (1992) 26. 


\section{Tables}

\begin{tabular}{|c|c|c|c|}
\hline & $\mathrm{Na}$ & $\mathrm{Al}$ & $\mathrm{F}$ \\
\hline$\sigma_{\text {inc }}[$ barn $]$ & 1.62 & 0.0082 & 0.0008 \\
$\sigma_{\text {coh }}[$ barn] $]$ & 1.66 & 1.495 & 4.017 \\
$c$ & 0.3 & 0.1 & 0.6 \\
\hline
\end{tabular}

Table 1

Incoherent and coherent neutron scattering cross-sections [17] $\left(\sigma_{c o h}=4 \pi b_{c o h}^{2}\right)$, and concentrations of $\mathrm{Na}, \mathrm{Al}$ and $\mathrm{F}$ for the cryolite composition. 


\section{Figures}

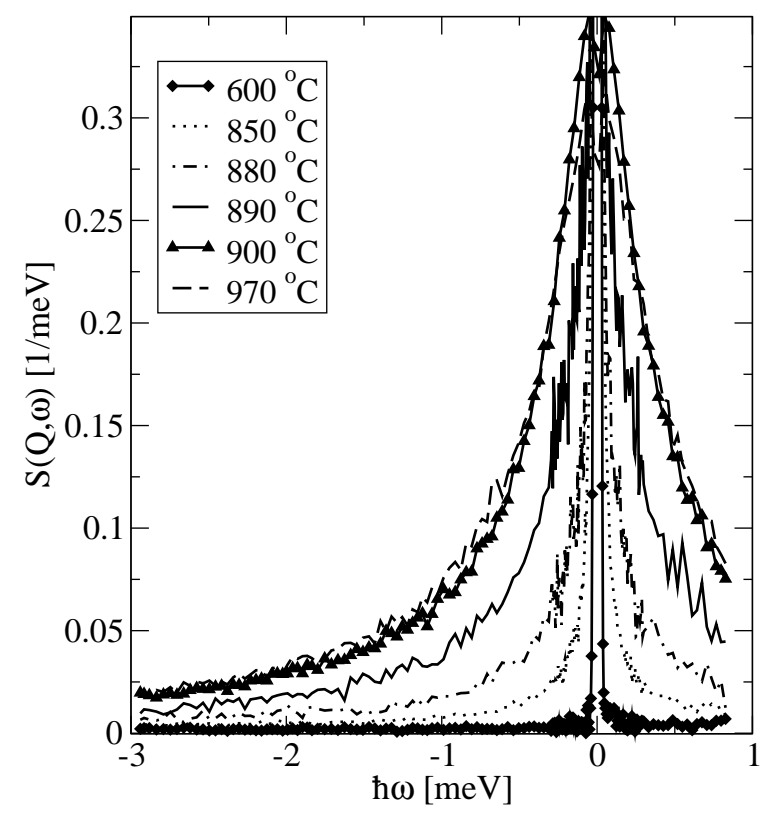

Fig. 1. Temperature dependence of the dynamic structure factor $S(Q, \omega)$ at constant $Q=1.0 \AA^{-1}$ using an incident neutron wavelength of $\lambda=7.6 \AA$.

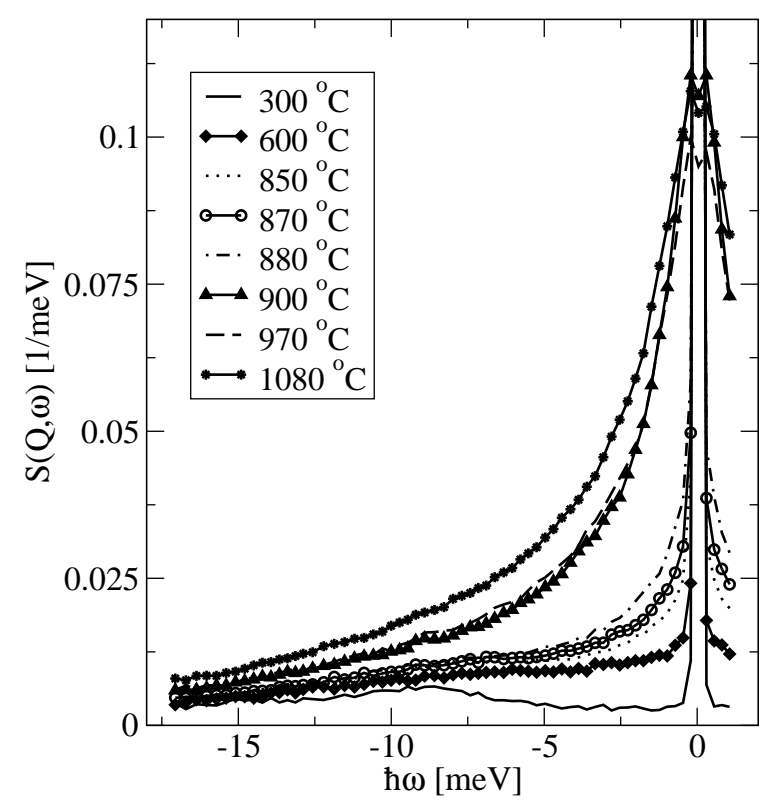

Fig. 2. Temperature dependence of the dynamic structure factor $S(Q, \omega)$ at constant $Q=2.0 \AA^{-1}$ using an incident neutron wavelength of $\lambda=5.0 \AA$. 


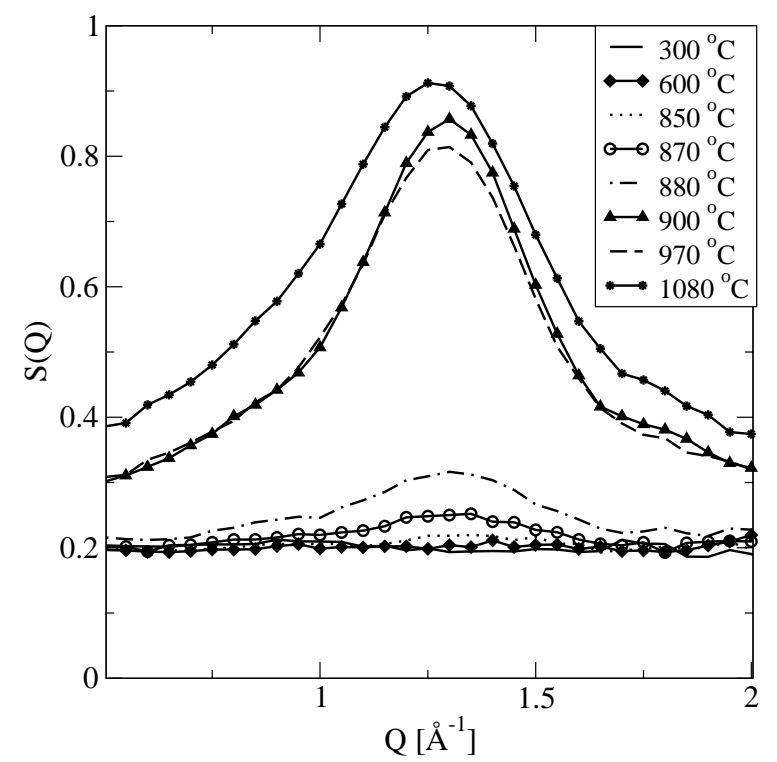

Fig. 3. Static structure factor, $S(Q)$, from integration over the measured $\hbar \omega$ range of $S(Q, \omega)$ as a function of $T(\lambda=5.0 \AA)$. A powder diffraction line at about $Q=1.3 \AA$ present in the crystal up to $T=880^{\circ} \mathrm{C}$ is several orders higher in intensity and has been omitted.

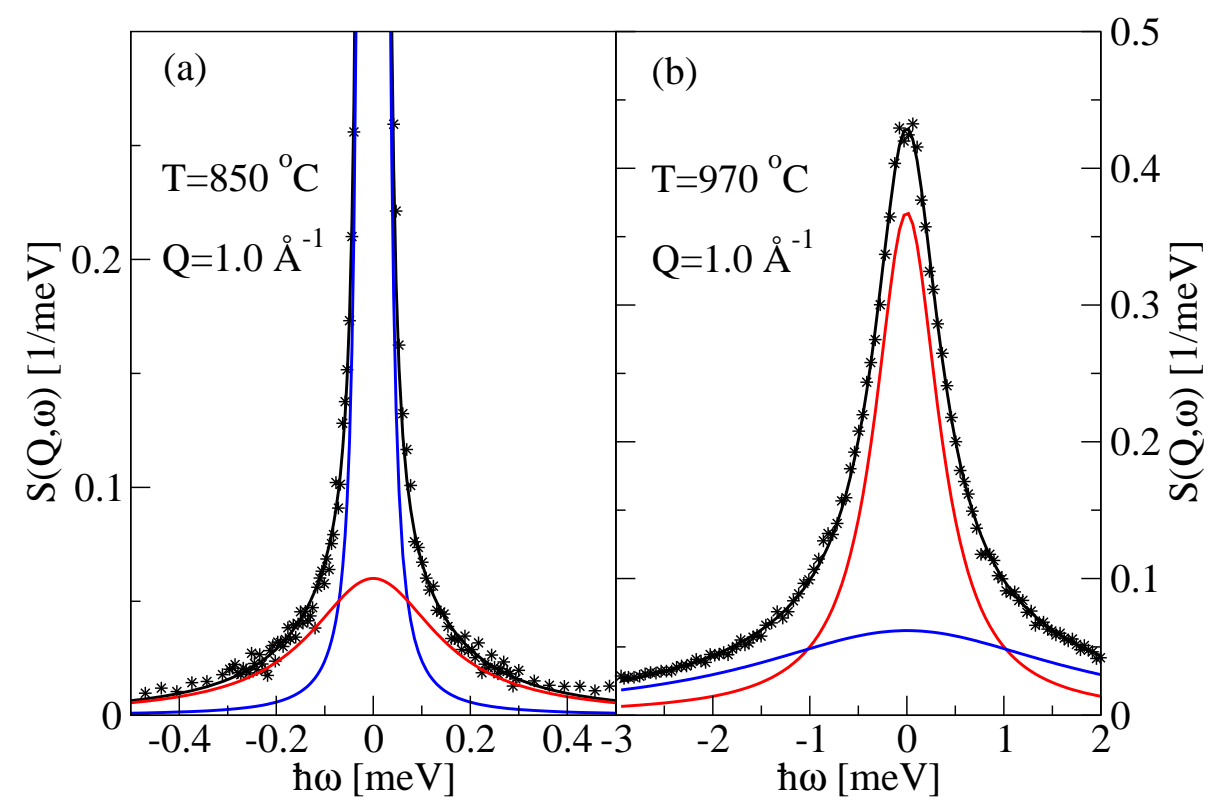

Fig. 4. Fit of $S(Q, \omega)$ at constant $Q=1.0 \AA^{-1}$ with two Lorentzians (lines) convoluted with the resolution function. The data (symbols) at $T=850{ }^{\circ} \mathrm{C}$ (a) have been obtained with $\lambda=7.6 \AA$ and at $T=970{ }^{\circ} \mathrm{C}$ (b) with $\lambda=5.0 \AA$. 


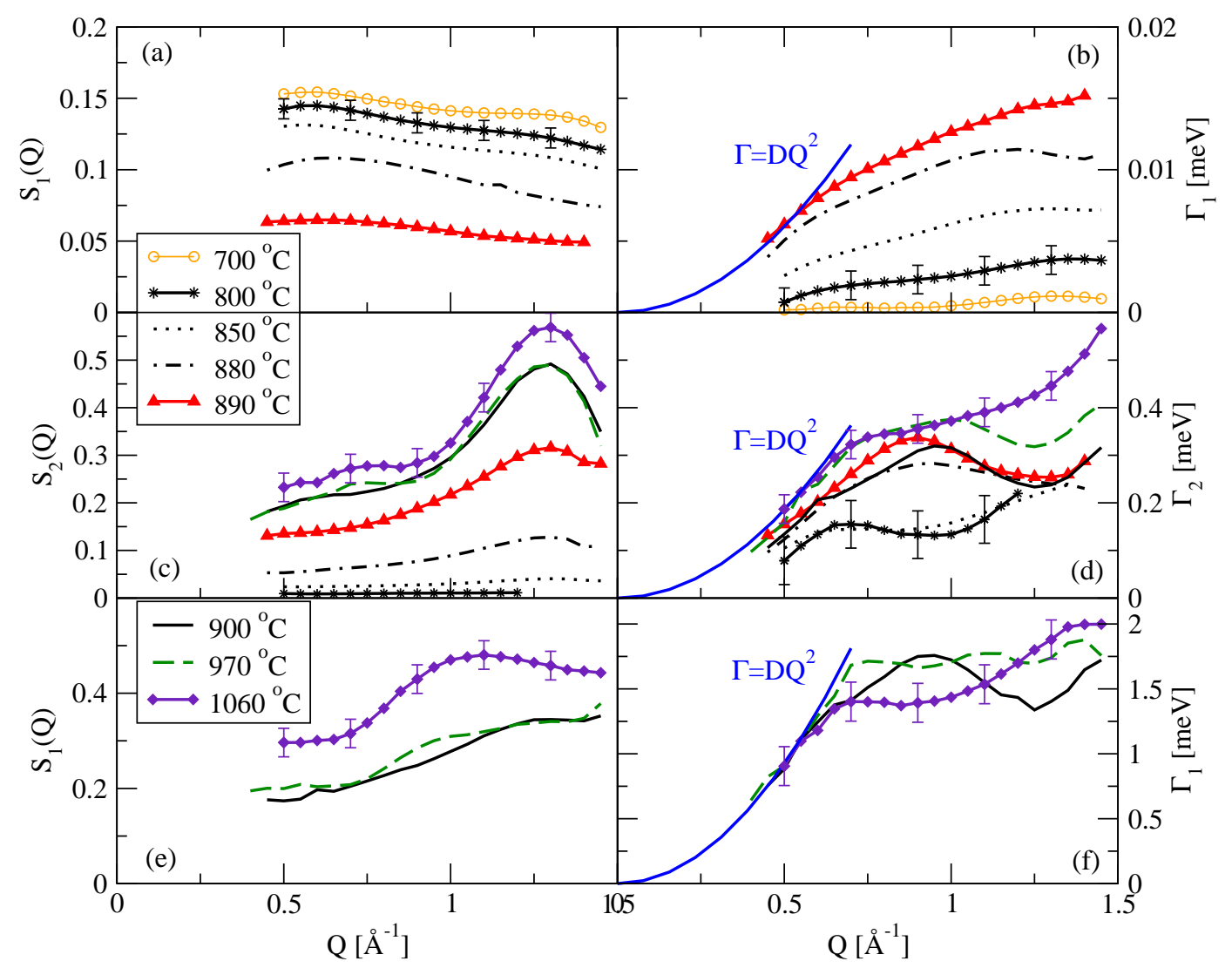

Fig. 5. Evolution of the fit parameters after fitting the dynamic structure factors, $S(Q, \omega)$, at constant $Q$ with two Lorentzians. $S_{1}(Q)$ and $S_{2}(Q)$ are the amplitudes, $\Gamma_{1}(Q)$ and $\Gamma_{2}(Q)$ the respective line widths. Different symbols represent different temperatures. The $Q^{2}$-dependence fitted to the line widths at low $Q$ is indicated. 


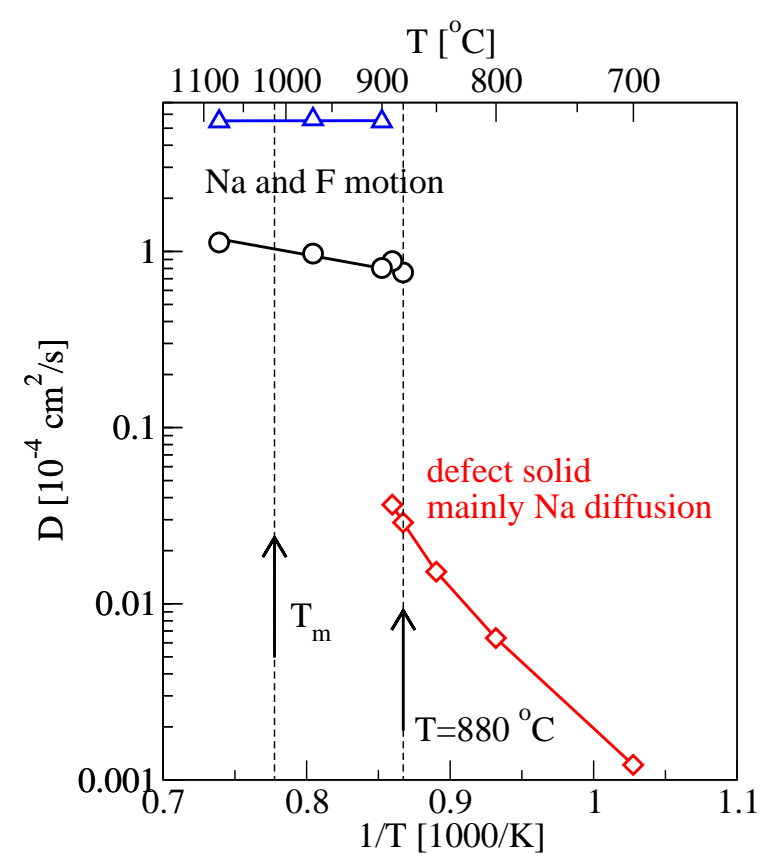

Fig. 6. Self-diffusion coefficients obtained from the $D Q^{2}$ dependence of the Lorentzian line widths at small $Q$. Below $880^{\circ} \mathrm{C}$, fluorine motion should be dominated by rotations and the contribution to the diffusivity is expected to be small.

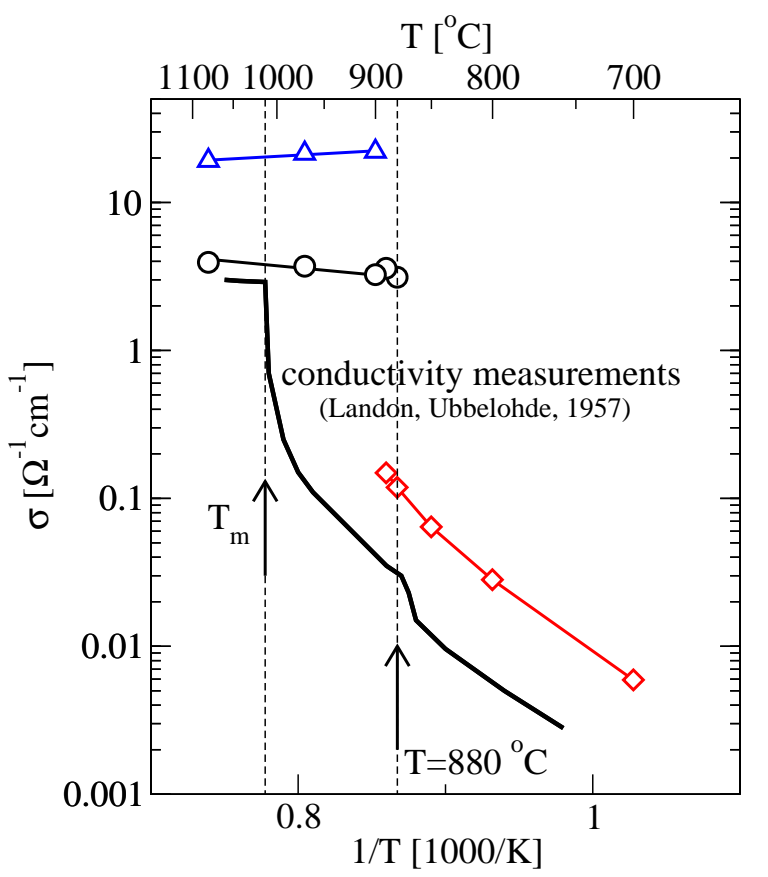

Fig. 7. Partial ionic conductivities derived from the self-diffusion coefficients as shown in Fig. 6 using the Nernst-Einstein equation assuming $D_{\sigma, i}=D_{i}$ (solid lines with symbols) The bold black solid line shows the results from electrical conductivity measurements by Landon and Ubbelohde [11]. 